\title{
Possible artemisinin-based combination therapy-resistant malaria in Nigeria: a report of three cases
}

\author{
Nnennaya Anthony Ajayi ${ }^{[1]}$ and Kingsley Nnanna Ukwaja ${ }^{[1]}$
}

[1]. Department of Internal Medicine, Federal Teaching Hospital Abakaliki, State of Ebonyi, Nigeria.

\begin{abstract}
Artemisinin-based combination therapy-resistant malaria is rare in Sub-Saharan Africa. The World Health Organization identifies monitoring and surveillance using day-3 parasitaemia post-treatment as the standard test for identifying suspected artemisinin resistance. We report three cases of early treatment failure due to possible artemisinin-based combination therapy-resistant Plasmodium falciparum malaria. All cases showed adequate clinical and parasitological responses to quinine. This study reveals a need to re-evaluate the quality and efficacy of artemisinin-based combination therapy agents in Nigeria and Sub-Saharan Africa.
\end{abstract}

Keywords: Malaria. Artemisinin-based combination therapy. Artemether-lumefantrine.

Early treatment failure. Resistance. Nigeria.

\section{INTRODUCTION}

The World Health Organization (WHO) currently recommends artemisinin-based combination therapy (ACT) as the first-line treatment for uncomplicated Plasmodium falciparum malaria in Sub-Saharan Africa (SSA) ${ }^{1}$. The combination consists of an artemisinin derivative with a rapid effect on parasite clearance and a longer-acting drug to prevent recrudescence. The massive reductions in malaria-related morbidity and mortality in regions of high endemicity in the last decade have been in part due to the effectiveness of the ACT regimen ${ }^{2}$. However, these successes are threatened by the emergence of artemisinin-resistant strains of Plasmodium falciparum from the Thai-Cambodian border ${ }^{3}$ and Thai-Myanmar border ${ }^{4}$. Indeed, artemisinin resistance is a major threat to global health, particularly in low- and middle-income countries (LMICs), in which the disease burden is highest, substandard or counterfeit ACT compounds are widely available, and systems for the monitoring and containment of resistance are inadequate ${ }^{5-7}$.

There is little existing knowledge regarding ACT-resistant malaria in many SSA countries, including Nigeria, and the most recent reports of ACT treatment failures were in travelers who had recently visited African countries ${ }^{8-10}$. Additionally, there have been no reports of delayed parasite clearance in routine therapeutic efficacy studies conducted in Africa $^{5}$. Thus, arguments for the presence of artemisinin resistance in Africa have been based solely on in vitro and/or molecular analyses

Address to: Dr. Kingsley N. Ukwaja. Department of Internal Medicine/Federal Teaching Hospital. 23433 Abakaliki, Ebonyi State, Nigeria.

Phone: 234080 3624-3196

e-mail: ukwajakingsley@yahoo.co.uk

Received 8 May 2013

Accepted 11 July 2013 of parasites collected from autochthonous patients or returning travellers ${ }^{4,5,9}$. However, standard in vitro tests are not reliable tools for monitoring artemisinin resistance ${ }^{3,5}$. In addition, none of the putative molecular markers for antimalarial drug resistance has been correlated with delayed clearance after treatment with artemisinin ${ }^{3}$. We recently encountered three cases of Plasmodium falciparum malaria that showed early treatment failure (ETF) of ACT and adequate clinical response to quinine.

\section{CASE REPORT}

\section{Case 1}

A 36-year-old female presented with a three-day history of high-grade fever with chills and a two-day history of productive cough associated with pleuritic chest pain and yellowness of the eyes. The patient is a known sickle cell disease patient. She had taken oral sulfadoxine-pyrimethamine and ciprofloxacin one day before presentation. On examination, she had a temperature of $39.5^{\circ} \mathrm{C}$ and was moderately pale, icteric, and mildly dehydrated. She had a respiratory rate of 44 cycles/minute, and her breath sounds were vesicular with coarse crackles in the right lower lung zone. Her pulse rate was 108 per minute and her blood pressure was $130 / 70 \mathrm{mmHg}$. Abdominal and neurologic examinations were unremarkable. The provisional diagnosis was hemolytic crisis precipitated by sepsis from community-acquired pneumonia and poorly treated malaria in a known sickle cell disease patient. Blood samples were collected for complete blood count (CBC), film for malaria parasites, serum electrolytes, urea, and creatinine, hepatic enzymes, and blood culture assays. Chest X-ray, urinalysis, and urine culture were also performed.

The patient received intravenous ceftriaxone, oral Coartem (artemether-lumefantrine), oral erythromycin, oral paracetamol, and hematinics. Her blood film showed Plasmodium falciparum parasitemia $(++)$, and her $\mathrm{CBC}$ showed anemia and leukocytosis (Table 1). Urinalysis revealed nitrate $(+)$ and proteinuria $(++)$. 
TABLE 1 - Complete blood counts of the three cases of possible ACT-resistant malaria at presentation.

\begin{tabular}{|c|c|c|c|c|}
\hline Variable & Case 1 & Case 2 & Case 3 & Reference value \\
\hline WBC count & 28 & 9.9 & 1.5 & $4-11 \times 10^{9}$ cells/liter \\
\hline Neutrophils & 68 & 78 & 22 & $40-70 \%$ \\
\hline Lymphocytes & 31 & 20 & 77 & $20-40 \%$ \\
\hline Monocytes & 0 & 0 & 0 & $2-10 \%$ \\
\hline Basophils & 0 & 0 & 0 & $0-1 \%$ \\
\hline Thrombocytes & 180 & 162 & 178 & $150-400 \times 10^{9} /$ liter \\
\hline Malaria parasite & $(++)$ & $(+++)$ & $(++)$ & positive $(+)$ / negative $(-)$ \\
\hline
\end{tabular}

ACT: artemisinin-based combination therapy; WBC: white blood cell.

Other requested investigations were unremarkable. After $72 \mathrm{~h}$ of treatment, the patient was still febrile (temperature $39.4^{\circ} \mathrm{C}$ ), though her cough and chest pain were subsiding. A repeat blood film showed the continued presence of malaria parasites. The patient was started on injections of quinine $(600 \mathrm{mg}$ in glucose infusion) every $8 \mathrm{~h}$ for three days, while the antibiotic treatment continued. Two days after starting the quinine, she showed a progressive reduction in temperature, and her fever resolved (temperature $37.1^{\circ} \mathrm{C}$ ) after $72 \mathrm{~h}$. Oral quinine and doxycycline were then administered for the next four and seven days, respectively. Repeat blood films after days 7 and 28 showed no malaria parasitemia.

\section{Case 2}

This patient was a 33-year-old female health worker who presented with a two-day history of fever, headache, and abdominal discomfort. The fever was high-grade, intermittent, with chills, and she suffered four episodes of vomiting one day before presentation. The patient had taken sulfadoxinepyrimethamine prior to presentation. On examination, her temperature was $39.2^{\circ} \mathrm{C}$, and her respiratory rate was 38 cycles/minute. Other examination findings were unremarkable. A provisional diagnosis of uncomplicated malaria and urinary tract infection was made. Blood film for plasmodium parasitemia, $\mathrm{CBC}$, urinalysis, urine culture; serum electrolytes, urea, and creatinine; and abdomino-pelvic ultrasound scan were performed. A blood smear showed severe Plasmodium falciparum parasitemia $(+++)$. Her CBC showed anemia with some neutrophilia (Table 1). Other requested investigations were unremarkable. She was given analgesics, intramuscular arteether, and oral amodiaquine for three days.

Three days later, her fever remained high (temperature $38.5^{\circ} \mathrm{C}$ ), and she became excessively weak. A repeat blood film showed persistent Plasmodium falciparum parasitemia $(++)$, and a diagnosis of severe malaria was considered. Intravenous artesunate was started. However, her clinical state deteriorated at 48 hours after commencing artesunate, and the fever and parasitemia persisted. A diagnosis of probable ACT-resistant malaria was made. Injections of quinine $(600 \mathrm{mg}$ infused every $8 \mathrm{~h}$ ) was begun. After two days on quinine, the patient showed a progressive reduction in temperature, which fell to $36.9^{\circ} \mathrm{C}$. The injectable quinine was changed to oral quinine, and she was discharged the next day. A repeat blood smear performed on days 7 and 28 after starting quinine were both negative for malaria parasitemia.

\section{Case 3}

A 54-year-old woman presented with a six-day history of high-grade fever and a four-day history of cough. She reported having five episodes of vomiting two days before presentation. The cough was associated with pleuritic chest pain and was productive, with yellowish sputum. On examination, her temperature was $38.9^{\circ} \mathrm{C}$, her respiratory rate was 48 cycles/ minute, and she had coarse crepitations on the left middle lung zone. The patient was managed for community-acquired pneumonia to rule out malaria. Investigations performed included the following: chest X-ray, sputum culture, sputum for acid-fast bacilli, CBC, blood film for malaria parasites, and serum electrolytes, urea, and creatinine. She was initially given oral amoxicillin-clavulanate and clarithromycin while awaiting results. The blood smear showed Plasmodium falciparum parasitemia (++). Her CBC showed severe anemia with leucopenia, neutropenia, and lymphocytosis (Table 1). The sputum culture yielded an alpha-hemolytic streptococcus sensitive to ofloxacin, ceftriaxone, and amoxicillin-clavulanate. Other requested results were unremarkable. The oral antibiotics were continued, and oral Coartem (artemether-lumefantrine) was started. The patient also received a transfusion of two units of fresh whole blood.

After $72 \mathrm{~h}$ of treatment, the patient still exhibited a fever (temperature of $38.1^{\circ} \mathrm{C}$ ), though her cough and chest pain were resolving. A repeat blood film showed Plasmodium falciparum parasitemia $(+)$. Antibiotics were continued. On the sixth day of treatment, the fever persisted, with a temperature of $37.8^{\circ} \mathrm{C}$, but the cough and chest findings had resolved. However, a blood film still showed Plasmodium falciparum parasitemia $(+)$; thus, a diagnosis of ACT-resistant malaria was considered. Oral quinine was given $(600 \mathrm{mg}$ every eight hours for seven 
days). The fever resolved within the next $48 \mathrm{~h}$, and the patient was discharged home the next day. Two blood films performed on days 14 and 28 after the initiation of quinine were negative for malaria parasites.

\section{DISCUSSION}

Although ACT-resistant malaria is rare in Africa, the WHO advocates increased monitoring and surveillance to identify and contain artemisinin resistance ${ }^{5}$. According to the WHO classification of malaria treatment outcomes ${ }^{11}$, these patients had ETF. This is particularly worrisome because the cases also fulfill the WHO definition for suspected artemisinin resistance, i.e., detectable peripheral parasitemia $72 \mathrm{~h}$ after starting antimalarial treatment ${ }^{5}$. Because the in vivo estimation of the parasite clearance rate from peripheral blood parasite counts is becoming the standard method used for the surveillance of artemisinin resistance ${ }^{3}$, it is necessary to use this method to maintain surveillance for ACT-resistant malaria.

Artemether-lumefantrine remains a very commonly used ACT for the treatment of uncomplicated Plasmodium falciparum malaria in SSA, and a recent pooled analysis showed a 28-day parasitological cure rate of $97 \%$ in malaria-endemic areas ${ }^{12}$. Late clinical failure (LCF) of artemether-lumefantrine has been reported in three travelers who became ill after visiting Sierra Leone $^{8}$, Tanzania ${ }^{9}$, and Congo ${ }^{10}$. Because of the lipophilic nature of lumefantrine, its bioavailability increases when taken with fatty meals ${ }^{9,10}$. Due to a lack of concurrent pharmacological evaluation, two previous reports speculated that sub-optimal lumefantrine concentrations were responsible for the observed $\mathrm{LCF}^{8,10}$. However, pharmacological studies and genotyping in one recent report showed adequate plasma concentrations of lumefantrine and indicated that the clinical failure was due to parasite recrudescence rather than recurrent infection?.

This is the first report from Nigeria showing ETF with artemether-lumefantrine. Two of the cases described received full doses of artemether-lumefantrine without good clinical response. Although the observed ETF could have been caused by the use of substandard/counterfeit artemether-lumefantrine compounds, several other patients with malaria received and responded to the same drug, and there are no current reports of in vivo resistance to artemether-lumefantrine in Nigeria. Furthermore, the second case described received and failed to respond to a combination of arteether and amodiaquine.
Moreover, all the cases showed adequate clinical and parasitological response to quinine ${ }^{11}$. Therefore, the ETF in the patients described may be due to ACT resistance.

This report highlights the need for closer monitoring and surveillance for AC-resistant Plasmodium falciparum malaria and calls for the urgent reassessment of the quality and efficacy of ACTs in Nigeria. Finally, there is a need to discover the genetic factors of the parasite that cause artemisinin resistance and to identify effective markers to monitor its spread in Nigeria and other $\mathrm{LMICs}^{3,4}$.

\section{REFERENCES}

1. World Health Organization (WHO). Guidelines for the treatment of malaria. Geneva: WHO; 2010. [Cited 2013 May 24]. Available at: http:// whqlibdoc.who.int/publications/2010/9789241547925_eng.pdf/.

2. World Health Organization (WHO). World Malaria Report 2012. Geneva: WHO; 2012.

3. Miotto O, Almagro-Garcia J, Manske M, Macinnis B, Campino S, Rockett $\mathrm{KA}$, et al. Multiple populations of artemisinin-resistant Plasmodium falciparum in Cambodia. Nat Genet 2013; 45:648-665.

4. Dondorp AM, Nosten F, Yi P, Das D, Phyo AP, Tarning J, et al. Artemisinin resistance in Plasmodium falciparum malaria. N Engl J Med 2009; 361:455-467

5. World Health Organisation (WHO). Update on artemisinin resistance - April 2012. Geneva: WHO; 2012. [Cited 2013 May 24] Available at: http://www.who.int/malaria/publications/atoz/arupdate042012.pdf/.

6. Braz RM, Duarte EC, Tauil PL. Epidemiology of malaria in the municipality of Cruzeiro do Sul, State of Acre, Brazil, in 2010: uses of a control chart at the local level. Rev Soc Bras Med Trop 2012; 45:526-529.

7. Campos PA, Valente B, Campos RB, Gonçalves L, Rosário VE, Varandas L, et al. Plasmodium falciparum infection in pregnant women attending antenatal care in Luanda, Angola. Rev Soc Bras Med Trop 2012; 45:369-374.

8. Mizuno Y, Kato Y, Kudo K, Kano S. First case of treatment failure of artemether-lumefantrine in a Japanese traveller with imported falciparum malaria. Jpn J Infect Dis 2009; 62:139-141.

9. Färnert A, Ursing J, Tolfvenstam T, Rono J, Karlsson L, Sparrelid E, et al. Artemether-lumefantrine treatment failure despite adequate lumefantrine day 7 concentration in a traveller with Plasmodium falciparum malaria after returning from Tanzania. Malar J 2012; 11:176.

10. Repetto EC, Traverso A, Giacomazzi CG. Possible clinical failure of artemether-lumefantrine in an Italian traveller with uncomplicated falciparum malaria. Mediterr J Hematol Infect Dis 2011; 3:e2011041.

11. World Health Organization (WHO). Methods for surveillance of antimalarial drug efficacy. Geneva: WHO; 2009.

12. Makanga M, Bassat Q, Falade CO, Premji ZG, Krudsood S, Hunt P, et al. Efficacy and safety of artemether-lumefantrine in the treatment of acute, uncomplicated Plasmodium falciparum malaria: a pooled analysis. Am J Trop Med Hyg 2011; 85:793-804. 